\title{
Mortality due to cancer treatment delay: systematic review and meta-analysis
}

\section{FAST TRACK}

\author{
Timothy P Hanna, ${ }^{1,2,3}$ Will D King, ${ }^{3}$ Stephane Thibodeau, ${ }^{2}$ Matthew Jalink, ${ }^{1,2}$ Gregory A Paulin, ${ }^{2}$ \\ Elizabeth Harvey-Jones, ${ }^{4}$ Dylan E O'Sullivan, ${ }^{3}$ Christopher M Booth, ${ }^{1,2,3,5}$ Richard Sullivan, ${ }^{6}$ \\ Ajay Aggarwal ${ }^{4,6,7}$
}

For numbered affiliations see end of the article.

Correspondence to: THanna tim.hanna@kingstonhsc.ca (or@HannaRadOnc on Twitter: ORCID 0000-0003-3228-6042)

Additional material is published online only. To view please visit the journal online.

Cite this as: $B M J$ 2020;371:m4087 http://dx.doi.org/10.1136/bmj.m4087

Accepted: 16 October 2020

\section{ABSTRACT}

OBJECTIVE

To quantify the association of cancer treatment delay and mortality for each four week increase in delay to inform cancer treatment pathways.

\section{DESIGN}

Systematic review and meta-analysis.

\section{DATA SOURCES}

Published studies in Medline from 1 January 2000 to 10 April 2020.

\section{ELIGIBILITY CRITERIA FOR SELECTING STUDIES}

Curative, neoadjuvant, and adjuvant indications for surgery, systemic treatment, or radiotherapy for cancers of the bladder, breast, colon, rectum, lung, cervix, and head and neck were included. The main outcome measure was the hazard ratio for overall survival for each four week delay for each indication. Delay was measured from diagnosis to first treatment, or from the completion of one treatment to the start of the next. The primary analysis only included high validity studies controlling for major prognostic factors. Hazard ratios were assumed to be log linear in relation to overall survival and were converted to an effect for each four week delay. Pooled effects were estimated using DerSimonian and Laird random effect models.

RESULTS

The review included 34 studies for 17 indications ( $n=1272681$ patients). No high validity data were found for five of the radiotherapy indications or for cervical cancer surgery. The association between delay and increased mortality was significant $(P<0.05)$ for 13 of 17 indications. Surgery findings were consistent, with a mortality risk for each four week delay of 1.06-

\section{WHAT IS ALREADY KNOWN ON THIS TOPIC}

Delay in the treatment of cancer can have adverse consequences on outcome Previous meta-analyses of high validity studies have found evidence supporting a continuous relation between delay and mortality or local control

Despite its foundational importance, we lack standardised estimates of the effect of treatment delay for most treatment indications

\section{WHAT THIS STUDY ADDS}

This systematic review considered seven major cancer types (bladder, breast, colon, rectum, lung, cervix, and head and neck) and three treatment modalities (surgery, systemic treatment, and radiotherapy)

The data consistently show that a four week treatment delay is associated with increased mortality; further mortality was reported with longer delays

Policies focused on minimising system level delays in cancer treatment initiation could improve population level survival outcomes

1.08 (eg, colectomy $1.06,95 \%$ confidence interval 1.01 to 1.12 ; breast surgery $1.08,1.03$ to 1.13 ). Estimates for systemic treatment varied (hazard ratio range 1.01-1.28). Radiotherapy estimates were for radical radiotherapy for head and neck cancer (hazard ratio $1.09,95 \%$ confidence interval 1.05 to 1.14 ), adjuvant radiotherapy after breast conserving surgery $(0.98,0.88$ to 1.09$)$, and cervix cancer adjuvant radiotherapy $(1.23,1.00$ to 1.50$)$. A sensitivity analysis of studies that had been excluded because of lack of information on comorbidities or functional status did not change the findings.

\section{CONCLUSIONS}

Cancer treatment delay is a problem in health systems worldwide. The impact of delay on mortality can now be quantified for prioritisation and modelling. Even a four week delay of cancer treatment is associated with increased mortality across surgical, systemic treatment, and radiotherapy indications for seven cancers. Policies focused on minimising system level delays to cancer treatment initiation could improve population level survival outcomes.

\section{Introduction}

Delay in the treatment of cancer can have adverse consequences on outcome. However, despite its foundational importance, we lack standardised estimates of the effect of treatment delay on survival for most treatment indications. Previous metaanalyses have found evidence supporting a continuous association between delay and mortality ${ }^{12}$ or local control. $^{3}$ A wide variation in reporting of delay estimates has limited meta-analysis. ${ }^{4}$ Understanding the impact of delay on mortality and other outcomes such as recurrence or financial impact on patients is essential to designing cancer care systems, pathways, and models of care that deliver affordable and equitable outcomes. ${ }^{5}$

The need for an in-depth understanding of the impact of treatment delay on outcomes has come sharply into focus during the coronavirus 2019 (covid-19) pandemic. Many countries have experienced deferral of elective cancer surgery and radiotherapy, and reductions in the use of systemic treatments $^{6} 7$ because systems have reassigned healthcare resources to pandemic preparedness. ${ }^{8}$ The lack of high quality data on the impact of deferred and delayed cancer treatment has meant that the impact of covid-19 lockdown measures on patterns of care and subsequent outcomes has not been robustly quantified. More broadly, in non-pandemic times, health systems have developed pathways and targets for intervals from 
the time of diagnosis to receipt of treatment within National Cancer Control Plan frameworks that do not have a strong empirical basis. ${ }^{9}$

Our analysis aims to provide robust evidence to guide national policy making, specifically the prioritisation and organisation of cancer services, by investigating the association between delays in receipt of cancer treatment and mortality. We considered seven common cancers and provide estimates on the impact of delay across all three curative modalities: surgery, systemic treatment, and radiotherapy delivered in the radical, neoadjuvant, and adjuvant setting.

\section{Methods \\ Population}

We investigated seven cancers that together represent $44 \%$ of all incident cancers globally ${ }^{10}$ : five common cancers (bladder, breast, colon, rectum, lung); cervical cancer, given its global importance as the fourth most common cancer diagnosis among women; and head and neck cancer (a major burden in middle income settings), for which there is an established association between delay and mortality. ${ }^{10}$ We selected these cancers by balancing representativeness with comprehensiveness. We also considered rectal and colon cancer separately given that radiotherapy is an integral part of treatment for rectal cancer but not colon cancer. Because of the generally indolent nature of prostate cancer (particularly for low and intermediate risk disease) compared with other cancers, and a preliminary review of the delay literature, this cancer was excluded because delays

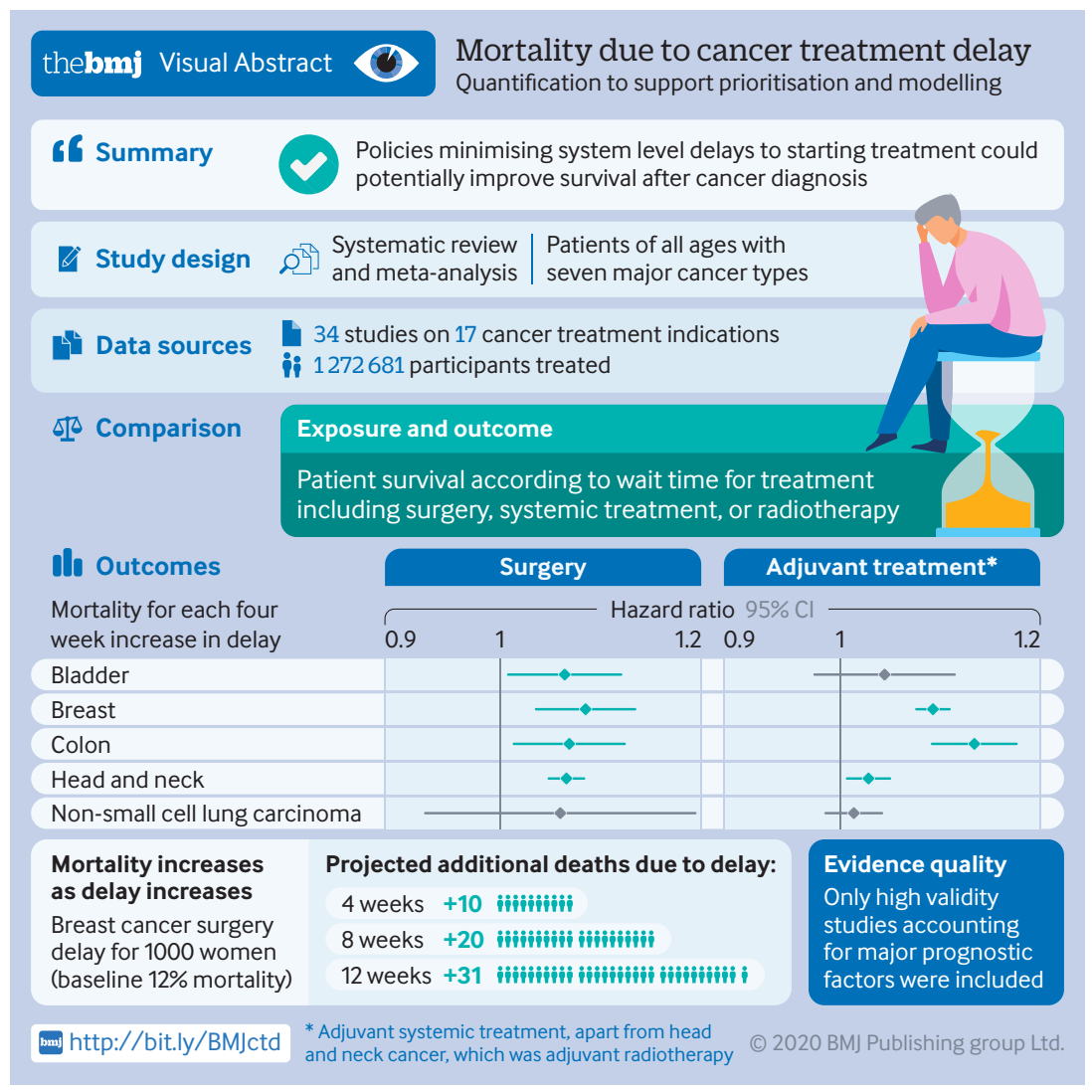

of the magnitude considered in our analysis were probably not associated with increased mortality.

\section{Exposure}

Treatment delay was defined as time from diagnosis to treatment for the first treatment (definitive surgery or radiation), and from time of surgery to treatment for adjuvant indications (chemotherapy or radiation after surgery). For neoadjuvant treatments (those delivered before primary curative therapy, eg, surgery), delay was defined as the time from diagnosis to the start of neoadjuvant treatment, or from the end of neoadjuvant treatment to time of surgery. Delay of curative treatments was investigated (surgery, systemic treatment, and radiotherapy).

\section{Outcome}

A hazard ratio for overall survival was estimated for each four week increase in delay. The hazard ratio represents the risk of death from any cause for patients experiencing the observed treatment delay compared with those treated without the delay.

\section{Systematic review}

We undertook a systematic review to identify high validity studies quantifying the impact of treatment delay on mortality. The PRISMA (preferred reporting items for systematic reviews and meta-analyses) guidelines were followed. ${ }^{11}$ We used Ovid Medline to carry out the search (appendix 1). To fully assess the validity of included studies, we did not search the literature for studies in abstract form only. Studies were limited to English language publications, from 2000 to present, and those reporting specifically on treatment delay and survival for the seven cancers being analysed. The year 2000 was selected to be comprehensive, while limiting reports to those reflective of contemporary practice as much as possible. We included studies if they specifically reported on the impact of delay for a well defined cancer indication. Studies that reported predominantly on patients receiving neoadjuvant treatments were excluded when evaluating the impact of treatment delay from diagnosis to definitive surgery. Studies that investigated the therapeutic benefit of intentional moderate delay between completion of neoadjuvant therapy for rectal cancer and surgery were excluded given potential confounding by indication. We did not exclude any studies based on design, except that the study needed to quantify the hazard ratio for overall survival because of treatment delay. The search was run on 10 April 2020, except for the bladder cancer search which was performed on 22 April 2020. Two reviewers screened abstracts by using Covidence systematic review software (Veritas Health Innovation, Melbourne, Australia). Searches of reference lists and Google were also performed.

We reviewed studies for evidence of control for major prognostic factors to assess risk of bias. The criteria used were consistent with those used by our group in other systematic reviews of delay and outcomes. ${ }^{1-3}$ 
Firstly we asked "was the distribution of the relevant prognostic factors adequately described in the groups of patients which were compared?” Relevant prognostic factors for all studies were considered to be age, stage, treatment description, and comorbidity or functional status. If no, the study was classified as not of high validity. If yes, we proceeded to the next question "Were the comparison groups balanced with respect to the relevant prognostic factors?” If yes, the study was classified as high validity. We qualitatively assessed the magnitude of observed differences, and the $\mathrm{P}$ value was considered when interpreting these differences. If no, we asked "Were the reported results appropriately adjusted for any differences in the relevant prognostic factors?" If yes, the study was classified as high validity; if no, the study was classified as not high validity. Only studies meeting these criteria were included for subsequent meta-analysis.

For some definitive indications (colon cancer, lung cancer, cervical cancer), it was possible that observed associations between treatment delay and risk of death were attenuated because patients with poorer outcomes might present more quickly with symptomatic disease through emergency or urgent referral pathways (often referred to as the waiting time paradox). ${ }^{12}$ To qualify as high validity, such studies were required to have also performed an analysis or subanalysis to investigate the impact of this factor in the observed associations. Similar to Neal and colleagues, this was defined as an analysis or subanalysis of patients clearly including or excluding patients with short diagnosis to treatment interval (eg, less than four weeks) or poor outcomes (eg, death within four to eight weeks of diagnosis). ${ }^{12}$

\section{Converting hazard ratios to four week delay estimates}

There was heterogeneous reporting of results, with time intervals reported as dichotomous, ordinal categories or as continuous variables. Results were converted to a common unit-hazard ratio for each four week delay with the assumption of a log linear relation across waiting times based on the findings of other meta-analyses..$^{1-3}$ A log linear relation predicts, for example, that patients waiting eight weeks rather than four weeks have a doubling in their risk of death. A unit of four weeks was chosen based on the magnitude of waiting times reported in the literature. We emphasise that the hazard ratio calculated in this study might be converted to shorter (eg, each week or each day) or longer units. Appendix 2 provides further information on the conversion of hazard ratios to each four week delay estimates or other units, and compares the log linear model to the linear model.

\section{Meta-analysis}

We obtained the summary hazard ratio estimate by pooling hazard ratios for each four week delay with inverse variance weighting in DerSimonian and Laird random effect models. Heterogeneity between studies was evaluated using the $\mathrm{I}^{2}$ test. We performed the statistical analysis using the $\mathrm{R}$ package metafor
(R Foundation for Statistical Computing, Vienna, Austria). We considered a two tailed $\mathrm{P}$ value less than 0.05 to be statistically significant. Publication bias was not tested given the small number of studies identified for each indication.

\section{Sensitivity analysis}

We undertook a post hoc sensitivity analysis to evaluate the impact of the stringent validity criteria on findings. Studies that had been excluded in the main analysis because of a lack of information on comorbidities or functional status were included in this analysis because other factors such as increasing age could be proxies for these.

\section{Patient and public involvement}

The research was informed by patient groups and cancer charities that were concerned about the impact of cancer treatment deferral and delays during the covid-19 pandemic.

\section{Results}

Our search identified 2543 articles for review (fig 1). ${ }^{11}$ After we added records identified through additional sources, and removed duplicates, 2843 records were screened. The primary reason for exclusion at the screening stage was lack of relevance to the study question. We obtained 275 articles to assess for eligibility. Of these, 241 were excluded, most commonly because they were not high validity studies $(n=100)$, they included the wrong patient population $(n=36)$, or the wrong study design $(n=26)$. This left 34 studies with unique populations for inclusion (fig 1 , table 1, table 2). ${ }^{13-46}$ These studies included 1272681 patients, with a samplesize ranging from 174 to 420792 (appendix 3). Twenty eight studies were population or registry based, and six were institutional reports. All studies were retrospective observational comparisons. Abstracted data on delay were dichotomous in eight, continuous in nine, and categorical in 17 studies. Waiting time data generally covered from three to four weeks, to 16 weeks (appendix 3). Appendix 3 presents the association between treatment delay and survival for individual studies. In addition to adjustments for age, stage, and comorbidity or functional status, 91\% of studies accounted for one or more socioeconomic variables in their analysis, $82 \%$ accounted for insurance status, $65 \%$ for year of treatment or year of diagnosis, and $88 \%$ for institutional or geographical factors (appendix 4). We did not find any high validity data for five radiotherapy indications or cervical cancer surgery (table 1, table 2).

Figure 2, figure 3, figure 4 show summary results for all indications, with pooled estimates displayed for treatment site combinations where more than one high validity study exists. The random effects models showed a consistent association of surgical delay with increased mortality, with all indications showing a hazard ratio for each four week delay of between 1.06 and 1.08 (6-8\% increased chance of death for each four week delay in treatment). For example, 


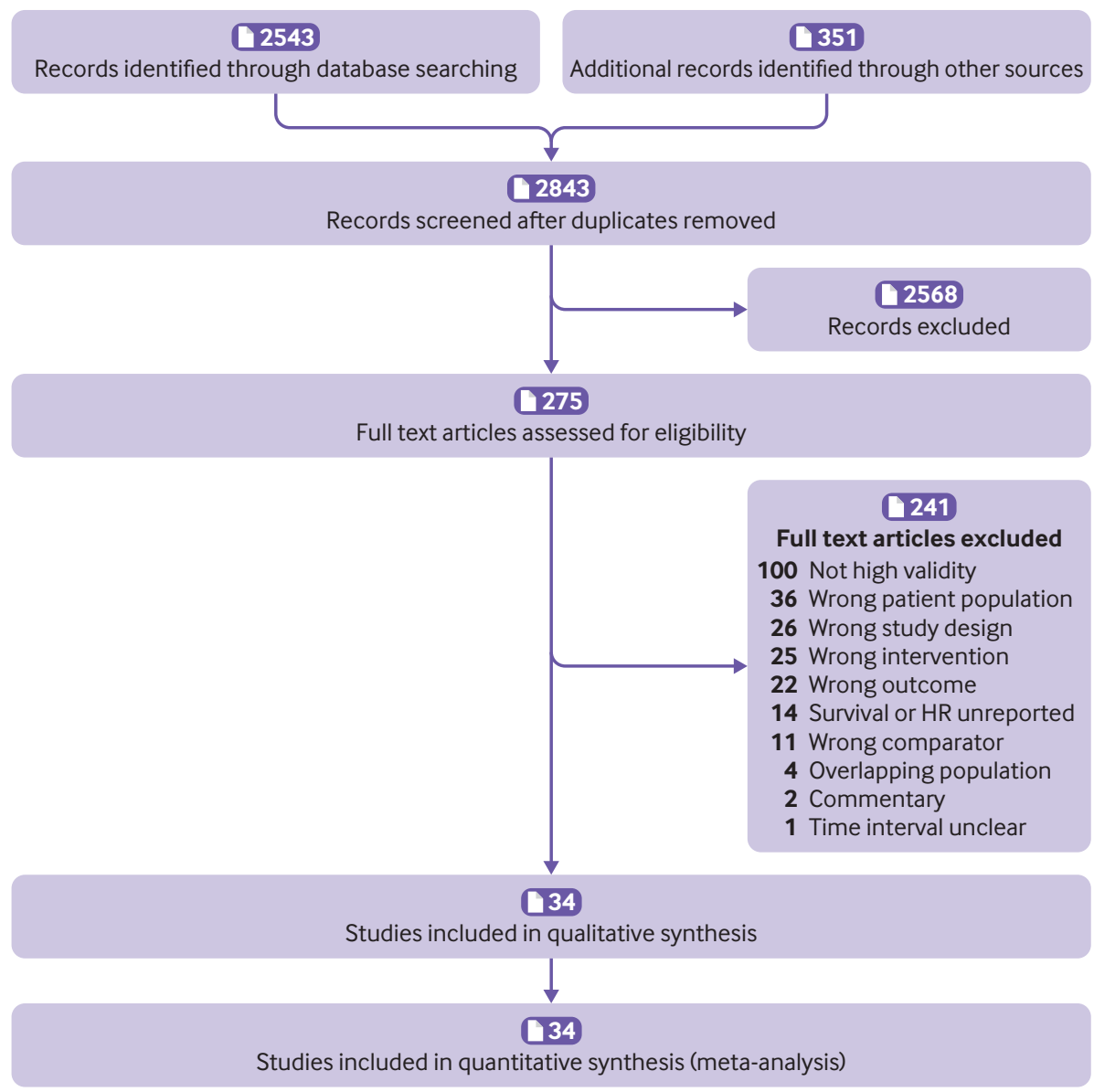

Fig 1 | PRISMA (preferred reporting items for systematic reviews and meta-analyses) 2009 flow diagram for systematic review of treatment delay and survival for curative surgery, systemic treatment, and radiotherapy for bladder, breast, colon, rectum, lung, cervix, and head and neck cancer. HR=hazard ratio

for head and neck surgery the hazard ratio was 1.06 (95\% confidence interval 1.04 to 1.08 ) and for breast partial or complete mastectomy the hazard ratio was 1.08 (1.03 to 1.13). The results for lung surgery were consistent with other sites, though not statistically significant (1.06, 0.93 to 1.19$)$.

Adjuvant and neoadjuvant systemic treatment indications varied more widely in effect (hazard ratio range 1.01-1.28). We observed significant associations for bladder neoadjuvant systemic treatment (hazard ratio $1.24,95 \%$ confidence interval 1.03 to 1.50 ), breast adjuvant (1.09, 1.07 to 1.11) and neoadjuvant systemic treatment $(1.28,1.05$ to 1.56$)$, and colon and rectal adjuvant chemotherapy $(1.13,1.09$ to 1.17$)$. Associations were non-significant for non-small cell lung cancer adjuvant chemotherapy (1.01, 0.99 to 1.04) and bladder adjuvant chemotherapy (1.04, 0.98 to 1.11 ).

High validity data on curative radiotherapy were limited, but supported a mortality impact of delay for head and neck cancer (eg, radical radiotherapy: $1.09,1.05$ to 1.14 ) and for cervical cancer adjuvant radiotherapy $(1.23,1.00$ to $1.50 ; \mathrm{P}=0.045)$. We found no significant effect for the single high validity study of adjuvant radiotherapy after breast conserving surgery $(0.98,0.88$ to 1.09$)$. No high validity studies were found for delay between diagnosis and start of neoadjuvant therapy for rectal cancer or for four other curative radiotherapy indications (table 2).

\section{Sensitivity analysis}

To evaluate the impact of our validity criteria on study findings, we undertook a sensitivity analysis and included studies that could be considered of borderline validity. For this analysis, we included 12 studies that were excluded in the primary analysis solely because of the lack of reporting or adjustment for comorbidity or functional status. We found little change in our estimates, except for breast cancer neoadjuvant systemic treatment (appendix 5).

\section{Discussion}

\section{Principal findings}

This analysis reports the impact of delay in curative treatment on the risk of death across the seven major tumour types: bladder, breast, colon, rectum, lung, cervix, and head and neck, and across all three major treatment modalities (surgery, systemic treatment, and radiotherapy). Across all three modalities, we found that a treatment delay of four weeks is associated 


\begin{tabular}{|c|c|c|c|c|c|c|}
\hline $\begin{array}{l}\text { Indication: } \\
\text { surgery }\end{array}$ & Source & Study design & Dataset (dates) & Median age (years) & Stage & Other study details \\
\hline \multirow[t]{3}{*}{ Bladder } & Chu $2019^{13}$ & $\begin{array}{l}\text { Retrospective observational } \\
\text { comparison }\end{array}$ & $\begin{array}{l}\text { SEER Medicare database } \\
(2004-2012)\end{array}$ & 75.2 (mean) & $\|$ & - \\
\hline & Gore $2009^{14}$ & $\begin{array}{l}\text { Retrospective observational } \\
\text { comparison }\end{array}$ & $\begin{array}{l}\text { SEER Medicare database } \\
(1992-2001)\end{array}$ & $\begin{array}{l}\leq 12 \text { weeks=73.8, >12 } \\
\text { weeks=73.6 (mean) }\end{array}$ & II & - \\
\hline & Kulkarni $2009^{15}$ & $\begin{array}{l}\text { Retrospective observational } \\
\text { comparison }\end{array}$ & $\begin{array}{l}\text { Ontario Cancer Registry } \\
(1992-2004)\end{array}$ & $\begin{array}{l}\leq 90 \text { days } 67.4, \\
>90 \text { days } 69.2 \text { (mean) }\end{array}$ & Tx, T0, Ta, Tis, T1-T4 & - \\
\hline \multirow[t]{5}{*}{ Breast } & Bleicher $2016^{16}$ & $\begin{array}{l}\text { Retrospective observational } \\
\text { comparison }\end{array}$ & $\begin{array}{l}\text { SEER Medicare database } \\
(1992-2009), \\
\text { NCDB (2003-2005) } \\
\text { databases }\end{array}$ & $\begin{array}{l}75.2 \text { (mean), } \\
60.3 \text { (mean) }\end{array}$ & $|-|||,|-|||$ & $\begin{array}{l}\text { Both cohorts were included } \\
\text { in meta-analysis as overlap } \\
\text { was limited owing to years } \\
\text { considered in two cohorts, } \\
\text { wider geographical population } \\
\text { coverage of NCDB with } \geq 18 \\
\text { years represented (SEER was } \\
\geq 66 \text { years) }\end{array}$ \\
\hline & Eaglehouse $2019^{17}$ & $\begin{array}{l}\text { Retrospective observational } \\
\text { comparison }\end{array}$ & $\begin{array}{l}\text { CCR, MDR databases } \\
(1998-2010)\end{array}$ & 54.5 (mean) & $|-| I \mid$ & - \\
\hline & Polverini $2016^{18}$ & $\begin{array}{l}\text { Retrospective observational } \\
\text { comparison }\end{array}$ & NCDB (2004-2012) & 59.4 (mean) & $|-| I \mid$ & - \\
\hline & Shin $2013^{19}$ & $\begin{array}{l}\text { Retrospective observational } \\
\text { comparison }\end{array}$ & KCCR database (2006) & 49.3 (mean) & $\begin{array}{l}\text { Local and regional } \\
\text { (SEER) }\end{array}$ & - \\
\hline & Mateo $2020^{20}$ & $\begin{array}{l}\text { Retrospective observational } \\
\text { comparison }\end{array}$ & NCDB (2010-2014) & NR & I-III & - \\
\hline \multirow[t]{2}{*}{ Colon } & Bagaria $2019^{21}$ & $\begin{array}{l}\text { Retrospective observational } \\
\text { comparison }\end{array}$ & $\begin{array}{l}\text { Multicentre, } \\
\text { US (1990-2012) }\end{array}$ & 71 (range 18-99) & I-IV (pathological) & - \\
\hline & Flemming $2017^{22}$ & $\begin{array}{l}\text { Retrospective observational } \\
\text { comparison }\end{array}$ & $\begin{array}{l}\text { OCR, CIHI DAD, OHIP } \\
\text { databases (2002-2008) }\end{array}$ & $71($ IQR 62-78) & I-IV (pathological) & - \\
\hline \multirow[t]{2}{*}{ NSCLC } & Kanarek $2014^{23}$ & $\begin{array}{l}\text { Retrospective observational } \\
\text { comparison }\end{array}$ & $\begin{array}{l}\text { Institutional US } \\
(2003-2009)\end{array}$ & $61 \% \geq 65$ & $1 \mathrm{~A}, 1 \mathrm{~B} / 2 \mathrm{~A}, 2 \mathrm{~B}$ & - \\
\hline & Samson $2015^{24}$ & $\begin{array}{l}\text { Retrospective observational } \\
\text { comparison }\end{array}$ & NCDB (1998-2010) & $\begin{array}{l}\text { <8 weeks: } 67.63( \pm 10.1) \\
\geq 8 \text { weeks: } 68.73( \pm 9.8) \\
(\text { mean }( \pm S D))\end{array}$ & I (clinical) & - \\
\hline Cervix & \multicolumn{6}{|c|}{ No high validity data found } \\
\hline \multirow[t]{2}{*}{$\begin{array}{l}\text { Head and } \\
\text { neck }\end{array}$} & Murphy $2016^{25}$ & $\begin{array}{l}\text { Retrospective observational } \\
\text { comparison }\end{array}$ & NCDB (2003-2005) & NR & I-IVB & $\begin{array}{l}\text { Oral tongue, oropharynx, } \\
\text { larynx, hypopharynx }\end{array}$ \\
\hline & Liao $2017^{26}$ & $\begin{array}{l}\text { Retrospective observational } \\
\text { comparison }\end{array}$ & $\begin{array}{l}\text { Taiwanese Cancer Registry } \\
\text { database (2004-2010) }\end{array}$ & 52.8 (mean) & I-IVB (clinical) & Oral cavity \\
\hline
\end{tabular}

$C C R=$ Department of Defence Central Cancer Registry; $C I H I D A D=C a n a d i a n$ Institute for Health Information Discharge Abstract Database; IQR=interquartile range; $K C C R=K o r e a n$ Central Cancer Registry; MDR=Military Health System Data Repository; NCDB=National Cancer Database (US); NR=not reported; NSCLC=non-small cell lung cancer; OCR=Ontario Cancer Registry; OHIP=Ontario Health Insurance Plan; SEER=Surveillance, Epidemiology, and End Results.

with an increase in the risk of death. For surgery, this is a $6-8 \%$ increase in the risk of death for every four week delay. This impact is even more marked for some radiotherapy and systemic indications, with a $9 \%$ and $13 \%$ increased risk of death for definitive head and neck radiotherapy and adjuvant systemic treatment for colorectal cancer, respectively. The one high validity study for breast cancer adjuvant radiotherapy did not show an effect, although a clear effect of delay on local control has been described (hazard ratio for each month of delay 1.08 , 95\% confidence interval 1.02 to 1.14 ); longer delays (eg, >20 weeks) have been associated with worse breast cancer specific survival. ${ }^{47} 48$

Policy implications and comparison to other studies Our analysis builds on the foundations of Mackillop and colleagues, who investigated the mortality impact per one month delay for radiotherapy indications (eg, head and neck, breast) and similarly for systemic treatment (adjuvant colon, breast). ${ }^{1-3} 47$ Our study provides a strong empirical basis for estimating the mortality impact of system level delays for different treatment modalities and cancers.

Delays of up to eight weeks and 12 weeks further increase the risk of death. For example, an eight week delay in breast cancer surgery would increase the risk of death by $17 \%$ (=1.08 $\left.8^{8 \text { weeks/4weeks }}\right)$ and a 12 week delay would increase the risk by $26 \%\left(=1.08^{12 \text { weeks/4weeks }}\right)$. Such figures translate into significant population level excess mortality. A surgical delay of 12 weeks for all patients with breast cancer for a year (eg, during covid-19 lockdown and recovery) would lead to 1400 excess deaths in the United Kingdom, 6100 in the United States, 700 in Canada, and 500 in Australia, assuming surgery is the first treatment in $83 \%$, and mortality without delay is $12 \% .^{10} 1649$ These results are sobering and suggest that the survival gained by minimising the time to initiation of treatment is of similar (and perhaps greater) magnitude of benefit as that seen with some novel therapeutic agents. ${ }^{50}$ Furthermore, our results do not consider the impact of treatment delay on local control rates, functional outcomes (eg, continence, swallowing), complications from more extensive treatments because of progression during delays, quality of life, ${ }^{51}$ or the greater economic burden because of higher direct care costs and productivity losses because of premature mortality and morbidity. ${ }^{52}$ Therefore, the impact of treatment delay is probably far greater for patients and society than that reflected in our results. 


\begin{tabular}{|c|c|c|c|c|c|c|}
\hline Indication & Source & Study design & Dataset (dates) & Median age (years) & Stage & Other study details \\
\hline \multicolumn{7}{|l|}{ Systemic treatment } \\
\hline $\begin{array}{l}\text { Neoadjuvant chemotherapy, } \\
\text { bladder }\end{array}$ & Chu $2019^{13}$ & $\begin{array}{l}\text { Retrospective observational } \\
\text { comparison }\end{array}$ & $\begin{array}{l}\text { SEER Medicare database } \\
(2004-2012)\end{array}$ & 72.9 (mean) & II & $\begin{array}{l}\text { Same study as Chu } \\
2019^{13} \text { bladder surgery }\end{array}$ \\
\hline \multirow[t]{2}{*}{$\begin{array}{l}\text { Adjuvant chemotherapy, } \\
\text { bladder }\end{array}$} & Corbett $2019^{27}$ & $\begin{array}{l}\text { Retrospective observational } \\
\text { comparison }\end{array}$ & NCDB (2006-2013) & NR & pT3-T4 or pN+ & - \\
\hline & Booth $2014^{28}$ & $\begin{array}{l}\text { Retrospective observational } \\
\text { comparison }\end{array}$ & OCR & $38 \%$ were $\geq 70$ & $\begin{array}{l}18 \%<\mathrm{T} 3,82 \% \\
\mathrm{~T} 3-\mathrm{T} 4,68 \% \\
\text { node positive }\end{array}$ & - \\
\hline $\begin{array}{l}\text { Neoadjuvant chemotherapy, } \\
\text { breast }\end{array}$ & Sanford $2016^{29}$ & $\begin{array}{l}\text { Retrospective observational } \\
\text { comparison }\end{array}$ & $\begin{array}{l}\text { Research database at } \\
\text { University of Texas } \\
(1995-2007)\end{array}$ & 50 (range $24-83$ ) & I-III (clinical) & $\begin{array}{l}\text { Time from end of } \\
\text { neoadjuvant } \\
\text { chemotherapy to surgery }\end{array}$ \\
\hline \multirow[t]{3}{*}{$\begin{array}{l}\text { Adjuvant chemotherapy, } \\
\text { breast }\end{array}$} & Gagliato $2014^{30}$ & $\begin{array}{l}\text { Retrospective observational } \\
\text { comparison }\end{array}$ & $\begin{array}{l}\text { MD Anderson Cancer } \\
\text { Center institutional } \\
\text { database. (1997-2011) }\end{array}$ & 50 (range 19-85) & $|-|||$ & - \\
\hline & Mateo $2020^{20}$ & $\begin{array}{l}\text { Retrospective observational } \\
\text { comparison }\end{array}$ & NCDB (2010-2014) & NR & $|-|||$ & - \\
\hline & Hershman $2006^{31}$ & $\begin{array}{l}\text { Retrospective observational } \\
\text { comparison }\end{array}$ & $\begin{array}{l}\text { SEER Medicare database } \\
(1992-1999)\end{array}$ & NR & $|-| \mid$ & - \\
\hline \multirow[t]{7}{*}{$\begin{array}{l}\text { Adjuvant chemotherapy, } \\
\text { colon, rectum }\end{array}$} & Hershman $2006^{32}$ & $\begin{array}{l}\text { Retrospective observational } \\
\text { comparison }\end{array}$ & $\begin{array}{l}\text { SEER Medicare database } \\
(1992-1999)\end{array}$ & NR & III Colon & - \\
\hline & Cheung $2009^{33}$ & $\begin{array}{l}\text { Retrospective observational } \\
\text { comparison }\end{array}$ & $\begin{array}{l}\text { SEER Medicare database } \\
(1991-2002)\end{array}$ & $\begin{array}{l}73.3 \\
(I Q R \text { 69.8-77.4) }\end{array}$ & II- III Rectal & - \\
\hline & Bayraktar $2011^{34}$ & $\begin{array}{l}\text { Retrospective observational } \\
\text { comparison }\end{array}$ & $\begin{array}{l}\text { Jackson Memorial Hospital } \\
\text { and University of Miami } \\
\text { Sylvester Comprehensive } \\
\text { Cancer Center (2000- } \\
\text { 2008) }\end{array}$ & $\begin{array}{l}55.7 \pm 1.1 \text { for } \leq 60 \\
\text { days and } 56.9 \pm 1.8 \\
\text { for }>60 \text { days } \\
(\text { mean } \pm \text { SE) }\end{array}$ & II-III Colon & - \\
\hline & Lima $2011^{35}$ & $\begin{array}{l}\text { Retrospective observational } \\
\text { comparison }\end{array}$ & $\begin{array}{l}\text { Alberta Cancer Registry, } \\
\text { ambulatory care } \\
\text { classification system, } \\
\text { discharge abstract } \\
\text { database (2000-2005) }\end{array}$ & NR & III Colon & - \\
\hline & Becerra $2017^{36}$ & $\begin{array}{l}\text { Retrospective observational } \\
\text { comparison }\end{array}$ & $\begin{array}{l}\text { New York State Registry, } \\
\text { SPARCS (2004-2009) }\end{array}$ & NR & III Colon & - \\
\hline & Xu $2014^{38}$ & $\begin{array}{l}\text { Retrospective observational } \\
\text { comparison }\end{array}$ & $\begin{array}{l}\text { SEER Medicare database } \\
(1992-2005)\end{array}$ & $\begin{array}{l}73.6 \\
(\text { IQR 69.8-77.6) }\end{array}$ & II Colon & - \\
\hline & Massarweh $2015^{39}$ & $\begin{array}{l}\text { Retrospective observational } \\
\text { comparison }\end{array}$ & NCDB (2003-2010) & $\begin{array}{l}60.8( \pm 11.6) \\
(\text { mean }( \pm S D))\end{array}$ & III Colon & - \\
\hline \multirow[t]{2}{*}{$\begin{array}{l}\text { Adjuvant chemotherapy, } \\
\text { NSCLC }\end{array}$} & Booth $2013^{40}$ & $\begin{array}{l}\text { Retrospective observational } \\
\text { comparison }\end{array}$ & OCR (2004-2006) & $\begin{array}{l}62(28-85) \\
(\text { mean (range)) }\end{array}$ & $\begin{array}{l}\text { I-IV } \\
\text { (pathological) }\end{array}$ & - \\
\hline & Salazar $2017^{41}$ & $\begin{array}{l}\text { Retrospective observational } \\
\text { comparison }\end{array}$ & NCDB (2004-2012) & 64 (IQR 57-70) & $\begin{array}{l}\text { I-III } \\
\text { (pathological) }\end{array}$ & - \\
\hline \multicolumn{7}{|l|}{ Radiotherapy } \\
\hline $\begin{array}{l}\text { Definitive radiotherapy/ } \\
\text { neoadjuvant, bladder }\end{array}$ & $\begin{array}{l}\text { No high validity } \\
\text { data found }\end{array}$ & & & & & \\
\hline $\begin{array}{l}\text { Adjuvant radiotherapy, post } \\
\text { breast conserving surgery }\end{array}$ & $\begin{array}{l}\text { Hébert-Croteau } \\
2004^{42}\end{array}$ & $\begin{array}{l}\text { Retrospective observational } \\
\text { comparison }\end{array}$ & $\begin{array}{l}\text { Random population based } \\
\text { sample of five regions of } \\
\text { Quebec, Canada for periods } \\
\text { covering } 1988-1994\end{array}$ & NR & $|-| \mid$ & - \\
\hline $\begin{array}{l}\text { Neoadjuvant (chemo)radiation, } \\
\text { rectum* }\end{array}$ & $\begin{array}{l}\text { No high validity } \\
\text { data found }\end{array}$ & & & & & \\
\hline $\begin{array}{l}\text { NSCLC, stage III } \\
\text { chemoradiation }\end{array}$ & $\begin{array}{l}\text { No high validity } \\
\text { data found }\end{array}$ & & & & & \\
\hline $\begin{array}{l}\text { SCLC, limited stage } \\
\text { chemoradiation }\end{array}$ & $\begin{array}{l}\text { No high validity } \\
\text { data found }\end{array}$ & & & & & \\
\hline $\begin{array}{l}\text { Adjuvant } \\
\text { chemoradiation, cervix }\end{array}$ & Jhawar $2017^{43}$ & $\begin{array}{l}\text { Retrospective observational } \\
\text { comparison }\end{array}$ & NCDB (2004-2013) & $46($ IQR 38-56) & $|\mathrm{B} 1-||| \mathrm{B}$ & $\begin{array}{l}\text { No stratified wait } \\
\text { group table but } \\
\text { adjusted analysis }\end{array}$ \\
\hline $\begin{array}{l}\text { Definitive chemoradiation, } \\
\text { cervix }\end{array}$ & $\begin{array}{l}\text { No high validity } \\
\text { data found }\end{array}$ & & & & & \\
\hline $\begin{array}{l}\text { Radical chemoradiation, } \\
\text { head and neck }\end{array}$ & Sharma $2016^{44}$ & $\begin{array}{l}\text { Retrospective observational } \\
\text { comparison }\end{array}$ & NCDB (2003-2006) & $\begin{array}{l}57.6(9.9) \\
(\text { mean }(S D))\end{array}$ & $\begin{array}{l}\text { III-IV (clinical, } \\
\text { non-metastatic) }\end{array}$ & $\begin{array}{l}\text { Oropharynx } \\
\text { chemoradiation }\end{array}$ \\
\hline $\begin{array}{l}\text { Adjuvant (chemo)radiation, } \\
\text { head and neck }\end{array}$ & Harris $2018^{45}$ & $\begin{array}{l}\text { Retrospective observational } \\
\text { comparison }\end{array}$ & NCDB (2004-2013) & $\begin{array}{l}59(10.9) \\
(\text { mean }(S D))\end{array}$ & $\begin{array}{l}\text { III-IV (non- } \\
\text { metastatic) }\end{array}$ & $\begin{array}{l}\text { Interaction between } \\
\text { subsite and outcome } \\
\text { observed }\end{array}$ \\
\hline $\begin{array}{l}\text { Radical (chemo)radiation, } \\
\text { nasopharyngeal carcinoma }\end{array}$ & Chen $2016^{46}$ & $\begin{array}{l}\text { Retrospective observational } \\
\text { comparison }\end{array}$ & $\begin{array}{l}\text { Sun Yat-Sen University } \\
\text { Cancer Center, institutional } \\
\text { series (2009-2012) }\end{array}$ & $\begin{array}{l}\text { NR, } 45 \% \leq 45 \\
\text { (primary cohort) }\end{array}$ & $\begin{array}{l}\text { I-IV (non- } \\
\text { metastatic) }\end{array}$ & $\begin{array}{l}99.6 \% \text { World Health } \\
\text { Organization histology } \\
\text { type II/III, treated with IMRT }\end{array}$ \\
\hline
\end{tabular}

IMRT=intensity modulated radiation therapy; IQR=interquartile range; NCDB=National Cancer Database (US); NR=not reported; NSCLC=non-small cell lung cancer; OCR=Ontario Cancer Registry; SCLC=small cell lung cancer; SD=standard deviation; SE=standard error; SEER=Surveillance, Epidemiology, and End Results; SPARCS=Statewide Planning and Research Cooperative System.

*Delay studies primarily investigating therapeutic benefit of usually short delay between completion of neoadjuvant treatment and surgery for rectal cancer are excepted. No high validity studies investigating time from diagnosis to start of neoadjuvant therapy were found for rectal cancer. 


\begin{tabular}{|c|c|c|}
\hline Study & $\begin{array}{l}\text { Hazard ratio } \\
(95 \% \mathrm{Cl})\end{array}$ & $\begin{array}{c}\text { Hazard ratio } \\
(95 \% \mathrm{Cl})\end{array}$ \\
\hline \multicolumn{3}{|l|}{ Bladder } \\
\hline Chu 2019 & $\longrightarrow \longrightarrow$ & $1.12(1.01$ to 1.25$)$ \\
\hline Gore 2009 & $\rightarrow \bullet$ & 1.09 (1.01 to 1.17$)$ \\
\hline Kulkarni 2009 & & $1.03(1.00$ to 1.06$)$ \\
\hline RE model: $Q=4.26 ; d f=2, P=0.12 ; l^{2}=53.0 \%$ & - & $1.06(1.01$ to 1.12$)$ \\
\hline \multicolumn{3}{|l|}{ Breast } \\
\hline Mateo 2020 & $\leftrightarrow$ & 1.09 (1.07 to 1.12$)$ \\
\hline Eaglehouse 2019 & 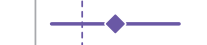 & 1.14 (1.02 to 1.27$)$ \\
\hline Bleicher (NCBD) 2016 & - & 1.09 (1.07 to 1.12$)$ \\
\hline Bleicher (SEER) 2016 & & $1.08(1.05$ to 1.12$)$ \\
\hline Polverini 2016 & $\diamond$ & 1.01 (1.01 to 1.02$)$ \\
\hline Shin 2013 & & $1.12(1.00$ to 1.25$)$ \\
\hline RE model: $Q=84.79 ; d f=5, P=0.00 ; I^{2}=94.1 \%$ & $\dot{i}$ & $1.08(1.03$ to 1.13$)$ \\
\hline \multicolumn{3}{|l|}{ Colon } \\
\hline Bagaria 2019 & $\longrightarrow-$ & $1.08(1.01$ to 1.15$)$ \\
\hline Flemming 2017 & - & 1.04 (0.95 to 1.13$)$ \\
\hline RE model: $Q=0.50 ; d f=1, P=0.48 ; l^{2}=0 \%$ & $\dot{i}$ & $1.06(1.01$ to 1.12$)$ \\
\hline \multicolumn{3}{|l|}{ Head and neck } \\
\hline Liao 2017 & $\leftrightarrow$ & 1.06 (1.04 to 1.09$)$ \\
\hline Murphy 2016 & $\rightarrow$ & $1.06(1.04$ to 1.09$)$ \\
\hline RE model: $Q=0.00 ; d f=1, P=0.95 ; I^{2}=0 \%$ & 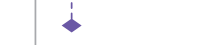 & $1.06(1.04$ to 1.08$)$ \\
\hline \multicolumn{3}{|l|}{ NSCLC } \\
\hline Samson 2015 & $\Rightarrow$ & $1.02(1.00$ to 1.03$)$ \\
\hline Kanarek 2014 & 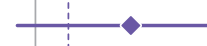 & $1.17(0.97$ to 1.41$)$ \\
\hline \multicolumn{3}{|l|}{ RE model: $Q=2.16 ; d f=1, P=0.14 ; I^{2}=53.8 \%$} \\
\hline 0.8 & 1.25 & \\
\hline
\end{tabular}

Fig 2 | Forest plot and pooled hazard ratios for association of each four week delay in surgery and overall survival by cancer site. Small purple diamonds represent the hazard ratio for each study and whiskers represent $95 \%$ confidence interval. Large purple diamonds represent summary effect estimates with the centre being the estimate and the ends representing $95 \%$ confidence intervals. NSCLC=non-small cell lung cancer

Treatment delays could be due to patient factors (eg, need for cardiac workup, postoperative wound infection), disease factors (eg, need for additional imaging investigations), or system factors (eg, waiting for an operating room date, a central line insertion, or a specialist consultation). The main purpose of this discussion is to highlight the need to minimise system level delays. We strongly emphasise that patients should not start surgery, systemic treatment, or radiotherapy until they are medically fit to do so, and have completed appropriate investigations. We also acknowledge that for rectal cancer, for instance, an increasing body of evidence shows that deferral of surgery after radiotherapy might not confer a survival disadvantage for those having a complete response. ${ }^{53}$

A major finding from our study is the paucity of high quality data for several tumour specific indications for radiotherapy, including chemoradiation for nonsmall cell lung cancer and definitive cervical cancer treatment. Two high validity studies providing delay estimates across multiple treatment modalities for these tumour types suggest an impact of delay in treatment initiation in these settings (cervical cancer mortality for each four week delay: hazard ratio 1.04,
95\% confidence interval 1.02 to $1.07^{54}$; stage III nonsmall cell cancer: $1.03,1.01$ to 1.06$).{ }^{55}$ While the negative impact of treatment interruptions on survival outcomes is well documented for these tumours, ${ }^{56-58}$ evidence is insufficient about the exact impact of a delay in starting treatment, which given its importance, should be an urgent research priority.

The study results are timely in light of the current covid-19 pandemic. Internationally, some countries have released national guidance on prioritisation of surgical treatments for cancer, which do not appear to be supported by the results of this study. For example, at the beginning of the pandemic the UK NHS ${ }^{59}$ created a short term surgical prioritisation algorithm. Several indications were considered safe to be delayed by 10-12 weeks with no predicted impact on outcome, including all colorectal surgery. Therefore, our results can help to directly inform policy-we found that increasing the wait to surgery from six weeks to 12 weeks would increase the risk of death in this setting by $9 \%$.

We note that a delay of less than four weeks should not be justified as safe based on our findings. For example, our results suggest a $4 \%$ increased risk of death for a two week delay for breast cancer surgery $\left(1.08^{2 \text { weeks/4weeks; }}\right.$ 


\begin{tabular}{|c|c|c|}
\hline Study & $\begin{array}{c}\text { Hazard ratio } \\
(95 \% \mathrm{Cl})\end{array}$ & $\begin{array}{c}\text { Hazard ratio } \\
(95 \% \mathrm{Cl})\end{array}$ \\
\hline \multicolumn{3}{|l|}{ Bladder neoadjuvant } \\
\hline Chu 2019 & $\rightarrow$ & $1.24(1.03$ to 1.50$)$ \\
\hline \multicolumn{3}{|l|}{ Bladder adjuvant } \\
\hline Corbett 2019 & $\rightarrow-$ & $1.03(1.00$ to 1.06$)$ \\
\hline Booth 2014 & & $1.13(0.97$ to 1.31$)$ \\
\hline RE model: $Q=1.32 ; d f=1, P=0.25 ; I^{2}=24.2 \%$ & & 1.04 (0.98 to 1.11$)$ \\
\hline \multicolumn{3}{|l|}{ Breast neoadjuvant } \\
\hline Sanford 2016 & & $1.28(1.05$ to 1.56$)$ \\
\hline \multicolumn{3}{|l|}{ Breast adjuvant } \\
\hline Mateo 2020 & $\bullet$ & $1.09(1.08$ to 1.11$)$ \\
\hline Gagliato 2014 & $\longrightarrow-$ & $1.06(1.00$ to 1.12$)$ \\
\hline Hershman 2006 & $-\infty$ & $1.08(1.04$ to 1.12$)$ \\
\hline RE model: $Q=1.27 ; d f=2, P=0.53 ;\left.\right|^{2}=0 \%$ & 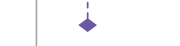 & $1.09(1.07$ to 1.11$)$ \\
\hline \multicolumn{3}{|l|}{ Colon and rectum adjuvant } \\
\hline Turner 2018 & $\diamond$ & 1.07 (1.06 to 1.08$)$ \\
\hline Beccera 2017 & $\stackrel{i}{-}-$ & $1.16(1.10$ to 1.22$)$ \\
\hline Massarweh 2015 & $\leftrightarrow$ & $1.13(1.10$ to 1.15$)$ \\
\hline Xu 2014 & & $1.35(1.22$ to 1.49$)$ \\
\hline Lima 2011 & $\bullet \frac{1}{1}$ & 1.06 (0.96 to 1.18$)$ \\
\hline Bayraktar 2011 & & 2.07 (1.02 to 4.19$)$ \\
\hline Cheung 2009 & $-\infty$ & $1.12(1.07$ to 1.16$)$ \\
\hline Hershman 2006 & - & 1.12 (1.07 to 1.18$)$ \\
\hline RE model: $Q=59.37 ; d f=7, P=0.00 ; I^{2}=88.2 \%$ & $<$ & $1.13(1.09$ to 1.17$)$ \\
\hline \multicolumn{3}{|l|}{ NSCLC adjuvant } \\
\hline Salazar 2017 & $\rightarrow$ & $1.01(0.99$ to 1.04$)$ \\
\hline Booth 2013 & i & $0.98(0.85$ to 1.13$)$ \\
\hline \multirow[t]{2}{*}{ RE model: $\mathrm{Q}=0.21 ; \mathrm{df}=1, \mathrm{P}=0.64 ; \mathrm{I}^{2}=0 \%$} & i & $1.01(0.99$ to 1.04$)$ \\
\hline & 1 & \\
\hline
\end{tabular}

Fig 3 | Forest plot and pooled hazard ratios for association of each four week delay in adjuvant and neoadjuvant systemic treatment and overall survival by cancer site. Small purple diamonds represent the hazard ratio for each study and whiskers represent $95 \%$ confidence interval. Large purple diamonds represent summary effect estimate with the centre being the estimate and the ends representing $95 \%$ confidence intervals. NSCLC=non-small cell lung cancer

appendix 2). Taken as a whole, these results suggest there is an urgent need to reconsider how we organise our cancer services. The prevailing paradigm has been around access to new treatments to improve outcomes, but from a system level, gains in survival might be achieved by prioritising efforts to minimise the time from cancer diagnosis to initiation of treatment from weeks to days. We acknowledge that treatment delays are multifactorial in cause and that patients should not start treatment before they are medically fit to do so, and have had completed all appropriate evaluations, however these data strongly support efforts to minimise system level delays. For example, national quality indicators around cancer waiting times from diagnosis to treatment are widely used across different health systems. In the UK NHS, current targets for the initiation of primary definitive treatment have been set at 31 days from the decision to treat date; this does not include the lag between receiving a diagnosis and having a surgical or radiation oncology consultation for treatment. ${ }^{9} 60$ At a population level, differences in lead times to treatment of even two or three weeks could be a factor in why survival outcomes differ across health systems and needs further investigation. However, these delays need to be balanced with the necessity to be medically fit for treatment. Additionally, potential opportunities for second opinions could result in more effective or appropriate care, especially where variation in practice or outcomes exist across providers.

Options for decreasing delay after diagnosis include increasing specialist workforce capacity through training initiatives or overcoming these challenges through technological developments. For example, automated treatment contouring and planning is increasingly standardised and reduces the radiotherapy preparation time to hours rather than days. ${ }^{61}$ Satellite centres might improve capacity for treating patients, as can reconfiguration of existing infrastructure to high volume super specialised services, or single entry models and team based care. ${ }^{62}$ Innovations in surgical technique could also minimise morbidity and reduce time to adjuvant therapy. ${ }^{6364}$ 


\begin{tabular}{|c|c|c|}
\hline Study & $\begin{array}{l}\text { Hazard ratio } \\
(95 \% \mathrm{Cl})\end{array}$ & $\begin{array}{l}\text { Hazard ratio } \\
(95 \% \mathrm{Cl})\end{array}$ \\
\hline \multicolumn{3}{|l|}{ Breast adjuvant } \\
\hline Hébert-Croteau 2004 & - & 0.98 (0.88 to 1.09 ) \\
\hline \multicolumn{3}{|l|}{ Cervix adjuvant } \\
\hline Jhawar 2017 & & 1.23 (1.00 to 1.50$)$ \\
\hline \multicolumn{3}{|l|}{ Head and neck radical } \\
\hline Sharma 2016 & $\longrightarrow-$ & 1.09 (1.05 to 1.14$)$ \\
\hline \multicolumn{3}{|l|}{ Head and neck adjuvant } \\
\hline Harris 2018 & $-\diamond-$ & 1.03 (1.01 to 1.05$)$ \\
\hline \multicolumn{3}{|l|}{ Nasopharyngeal } \\
\hline Chen 2016 & & 2.17 (1.03 to 4.57$)$ \\
\hline 0.8 & 1.25 & \\
\hline
\end{tabular}

Fig 4 | Forest plot of hazard ratios for association of each four week delay in radical and adjuvant radiotherapy and overall survival by cancer site. Purple diamonds represent the hazard ratio for each study and whiskers represent $95 \%$ confidence interval

\section{Strengths and limitations of this study}

Our study provides evidence on the association of treatment delay and mortality, covering seven cancer types and three treatment modalities. Our study was based on observational data, and we therefore restricted our sample to high validity studies given the biases inherent to this study design. Our approach provides high level evidence on system delay because randomised trials in this context are not appropriate or feasible.

The most fundamental limitation of our study is the risk of residual confounding. Patients with longer treatment delays could be destined to have inferior outcomes for reasons of comorbidity, treatment morbidity, or performance status. In evaluating the validity of our findings, we note the coherence of overall mortality and cancer specific endpoints (local control, cancer specific survival, disease-free survival) for all past meta-analyses of high validity studies. ${ }^{1-347}$ We also note major detrimental effects of prolonged waiting times on cancer specific survival outcomes in 13 of 15 studies included in our meta-analysis that reported cancer survival outcomes alongside overall survival. ${ }^{1416222829-36383946}$ These studies span seven treatment indications. Factors associated with medical status such as elements of socioeconomic status or insurance status might also be confounding factors; we found that $91 \%$ and $82 \%$ of identified studies accounted for these, respectively, though this does not completely rule out the possibility of residual confounding. Twenty five of 34 identified studies were from the USA, though no significant heterogeneity was detected compared with other countries.

Our findings cannot be directly applied to other cancer specific treatment indications, or to subgroups or single patients with treatment indications considered here. For example, limited evidence suggests that the impact of delay can vary according to stage, often with consistently greater mortality impact with earlier stage disease. ${ }^{16-18} 2555$ Additionally, our results can only be applied to the range of delay considered in the studies we evaluated. Given evidence derived from cancers representing almost half of all patients, the precautionary principle (acting to avoid or diminish harm in the face of scientific uncertainty) should be used when determining acceptable waiting times for treatment where data are limited. ${ }^{8}$ Too few studies were found for most indications to perform a risk of publication bias assessment with funnel plots. For previous meta-analyses where enough studies existed to do so, findings were not explained by publication bias. $^{12}$

We acknowledge that the assumption of a log linear relation between waiting time and mortality could be an oversimplification. This assumption was required to estimate per unit time mortality impact of delay from studies that use a variety of wait time representations. However, there is support for this assumption in the primary studies we used. A continuous exposure from nine primary studies assumed (log) linearity. Six studies undertook cubic spline analysis and the results are compatible with log linear effects with the range of wait times considered here (four weeks to 16 weeks). ${ }^{151921264145}$ Moreover, the previous metaanalyses by Biagi and colleagues and Raphael and colleagues suggest a reasonable fit of a log linear relation to delay. ${ }^{12}$ If a linear relation with delay exists (rather than log linear), the degree of difference in the two models is expected to be sufficiently small to allow use of a log linear model for the specific purposes of modelling the impact of delay on mortality between four and 16 weeks (appendix 2). We emphasise that assuming log linearity outside of the range of wait times used in this analysis is inappropriate. Our findings should also not be used to evaluate whether there is a minimal safe delay, or to estimate the impact of delay beyond 16 weeks.

Our results reflect the impact of delay on large and expectedly heterogeneous populations with varying risks of recurrence. Therefore, these estimates are best used at a policy and planning level for modelling, rather than for individual risk prediction. We also 
emphasise that few studies considered the impact of immortal time bias on delay; this could be done through a landmark analysis for survival. Patients that survived a longer wait might have less aggressive tumours, biasing the delay effect towards the null. Our findings could therefore underestimate the impact of delay on mortality.

\section{Conclusions}

A four week delay in treatment is associated with an increase in mortality across all common forms of cancer treatment, with longer delays being increasingly detrimental. In light of these results, policies focused on minimising system level delays in cancer treatment initiation could improve population level survival outcomes.

\section{AUTHOR AFFILIATIONS}

${ }^{1}$ Division of Cancer Care and Epidemiology, Cancer Research Institute at Queen's University, 10 Stuart Street, 2nd Level, Kingston, ON K7L3N6, Canada

${ }^{2}$ Department of Oncology, Queen's University, Kingston, ON, Canada

${ }^{3}$ Department of Public Health Sciences, Queen's University, Kingston, ON, Canada

4Department of Clinical Oncology, Guy’s \& St Thomas' NHS Trust, London, UK

${ }^{5}$ Department of Medicine, Queen's University, Kingston, ON, Canada

${ }^{6}$ Institute of Cancer Policy, King's College London, London, UK

${ }^{7}$ Department of Health Services Research and Policy, London School of Hygiene and Tropical Medicine, London, UK

Dr Patti Groome is acknowledged for providing thoughtful comments on the evaluation of confounding by indication.

Contributors: Concept and design: TPH, WDK, CMB, RS, AA. Acquisition, analysis, or interpretation of data: TPH, WDK, ST, MJ, GAP, EHJ, DEO, CMB, RS, AA. Statistical analysis: TPH, WDK, DEO. Drafting of the manuscript: TPH, AA, WDK. Critical revision of the manuscript for important intellectual content: TPH, WDK, ST, MJ, GAP, EHJ, DEO, CMB, RS, AA. Administrative, technical, or material support: TPH, WDK, AA. Supervision: TPH, WDK, AA. TPH had full access to all of the data in the study, takes responsibility for the integrity of the data and the accuracy of the data analysis, and is guarantor for this work. TPH is the corresponding author and attests that all listed authors meet authorship criteria and that no others meeting the criteria have been omitted.

Funding: There was no project specific funding for this work. TPH holds a research chair provided by the Ontario Institute for Cancer Research through funding provided by the Government of Ontario (\#IA-035). RS is funded through the UK Research and Innovation Economic and Social Research Council (ES/P010962/1). DEO is supported by an Ontario Graduate Scholarship and a Chen-Aronson Fellowship in Causes of Cancer. CMB is supported as the Canada Research Chair in Population Cancer Care. AA is supported by a National Institute for Health Research (NIHR) Advanced Fellowship (NIHR300599). The views expressed in this publication are those of the authors and not necessarily those of the NHS, the Government of Ontario, the National Institute for Health Research, or the Department of Health and Social Care.

Competing interests: All authors have completed the ICMJE uniform disclosure form at www.icmje.org/coi_disclosure.pdf and declare: no support from any organisation for the submitted work; unrestricted research funding for an unrelated project from Roche (TPH); no other relationships or activities that could appear to have influenced the submitted work.

Ethical approval: No ethical approval was required as this was a systematic review and meta-analysis of previously conducted studies. Data sharing: No additional data available.

The lead author affirms that the manuscript is an honest, accurate, and transparent account of the study being reported; that no important aspects of the study have been omitted; and that any discrepancies from the study as originally planned (and, if relevant, registered) have been explained.

Dissemination to participants and related patient and public communities: We plan to disseminate the results to patient organisations. Dissemination to study participants is not applicable.
Provenance and peer review: Not commissioned; externally peer reviewed.

This is an Open Access article distributed in accordance with the Creative Commons Attribution Non Commercial (CC BY-NC 4.0) license, which permits others to distribute, remix, adapt, build upon this work non-commercially, and license their derivative works on different terms, provided the original work is properly cited and the use is noncommercial. See: http://creativecommons.org/licenses/by-nc/4.0/.

1 Biagi JJ, Raphael MJ, Mackillop WJ, Kong W, King WD, Booth CM. Association between time to initiation of adjuvant chemotherapy and survival in colorectal cancer: a systematic review and meta-analysis. JAMA 2011;305:2335-42. doi:10.1001/jama.2011.749

2 Raphael MJ, Biagi JJ, Kong W, Mates M, Booth CM, Mackillop WJ. The relationship between time to initiation of adjuvant chemotherapy and survival in breast cancer: a systematic review and meta-analysis. Breast Cancer Res Treat 2016;160:17-28. doi:10.1007/s10549-016-3960-3

3 Chen Z, King W, Pearcey R, Kerba M, Mackillop WJ. The relationship between waiting time for radiotherapy and clinical outcomes: a systematic review of the literature. Radiother Oncol 2008;87:3-16. doi:10.1016/j.radonc.2007.11.016

4 Graboyes EM, Kompelli AR, Neskey DM, et al. Association of Treatment Delays With Survival for Patients With Head and Neck Cancer: A Systematic Review. JAMA Otolaryngol Head Neck Surg 2019;145:166-77. doi:10.1001/jamaoto.2018.2716

5 Institute of Medicine Committee on Quality of Health Care in America. Crossing the quality chasm: a new health system for the 21st century. National Academy Press, 2001

6 Sample I. More than $2 \mathrm{~m}$ operations cancelled as NHS fights Covid-19. The Guardian. 2020. https://www.theguardian.com/ society/2020/apr/26/more-than-two-million-operations-cancelledas-nhs-fights-covid-19

7 Harnett CE. Clearing surgery backlog will take at least 17 months and cost millions, province says. Times Colonist. 2020. https://www. timescolonist.com/news/local/clearing-surgery-backlog-will-take-atleast-17-months-and-cost-millions-province-says-1.24131366

8 Hanna TP, Evans GA, Booth CM. Cancer, COVID-19 and the precautionary principle: prioritizing treatment during a global pandemic. Nat Rev Clin Oncol 2020;17:268-70. doi:10.1038/ s41571-020-0362-6

9 NHS England. Achieving world-class cancer outcomes: a strategy for England 2015-2020 - progress report 2016-2017. 2017. https:// www.england.nhs.uk/publication/achieving-world-class-canceroutcomes/

10 Ferlay J, Ervik M, Lam F, et al. Global Cancer Observatory: Cancer Today. International Agency for Research on Cancer, 2018, https:// gco.iarc.fr/today

11 Moher D, Liberati A, Tetzlaff J, Altman DG, Group P. Preferred reporting items for systematic reviews and meta-analyses: the PRISMA statement. PLoS Med 2009;6:e1000097. doi:10.1371/journal.pmed.1000097

12 Neal RD, Tharmanathan P, France B, et al. Is increased time to diagnosis and treatment in symptomatic cancer associated with poorer outcomes? Systematic review. Br J Cancer 2015;112(Supp 1):S92-107. doi:10.1038/bjc.2015.48

13 Chu AT, Holt SK, Wright JL, Ramos JD, Grivas P, Yu EY, et al. Delays in radical cystectomy for muscle-invasive bladder cancer. Cancer 2019;125:2011-17. doi:10.1002/cncr.32048

14 Gore JL, Lai J, Setodji CM, et al. Mortality increases when radical cystectomy is delayed more than 12 weeks: results from a Surveillance, Epidemiology, and End Results-Medicare Analysis. Cancer 2009;115:988-96. doi:10.1002/cncr.24052

15 Kulkarni GS, Urbach DR, Austin PC, Fleshner NE, Laupacis A. Longer wait times increase overall mortality in patients with bladder cancer. Urol 2009;182:1318-24

16 Bleicher RJ, Ruth K, Sigurdson ER, et al. Time to Surgery and Breast Cancer Survival in the United States. JAMA Oncol 2016;2:330-9. doi:10.1001/jamaoncol.2015.4508

17 Eaglehouse YL, Georg MW, Shriver CD, Zhu K. Time-to-surgery and overall survival after breast cancer diagnosis in a universal health system. Breast Cancer Res Treat 2019;178:441-50. doi:10.1007/ s10549-019-05404-8

18 Polverini AC, Nelson RA, Marcinkowski E, et al. Time to treatment: measuring quality breast cancer care. Ann Surg Oncol 2016;23:3392-402. doi:10.1245/s10434-016-5486-7

19 Shin DW, Cho J, Kim SY, et al. Delay to curative surgery greater than 12 weeks is associated with increased mortality in patients with colorectal and breast cancer but not lung or thyroid cancer. Ann Surg Oncol 2013;20:2468-76. doi:10.1245/s10434-013-2957-y

20 Mateo AM, Mazor AM, Obeid E, et al. Time to surgery and the impact of delay in the non-neoadjuvant setting on triple-negative breas cancers and other phenotypes. Ann Surg Oncol 2020;27:1679-92. doi:10.1245/s10434-019-08050-y

21 Bagaria SP, Heckman MG, Diehl NN, Parker A, Wasif N. Delay to Colectomy and Survival for Patients Diagnosed with Colon Cancer. J Invest Surg 2019;32:350-7 
22 Flemming JA, Nanji S, Wei X, Webber C, Groome P, Booth CM. Association between the time to surgery and survival among patients with colon cancer: a population-based study. Eur / Surg Oncol 2017;43:1447-55. doi:10.1016/j.ejso.2017.04.014

23 Kanarek NF, Hooker CM, Mathieu L, Tsai H-L, Rudin CM, Herman JG, et al. Survival after community diagnosis of early-stage non-small cell lung cancer. Am J Med 2014;127:443-9. doi:10.1016/j. amjmed.2013.12.023

24 Samson P, Patel A, Garrett T, et al. Effects of delayed surgical resection on short-term and long-term outcomes in clinical stage I non-small cell lung cancer. Ann Thorac Surg 2015;99:1906-13. doi:10.1016/j.athoracsur.2015.02.022

25 Murphy CT, Galloway TJ, Handorf EA, Egleston BL, Wang LS, Mehra $R$, et al. Survival impact of increasing time to treatment initiation for patients with head and neck cancer in the United States. J Clin Oncol 2016:34:169-78.

26 Liao C-T, Chen H-N, Wen Y-W, Lee SR, Ng S-H, Liu T-W, et al. Association between the diagnosis-to-treatment interval and overall survival in Taiwanese patients with oral cavity squamous cell carcinoma. Eur J Cancer 2017;72:226-34.

27 Corbett CJ, Xia L, Mamtani R, Malkowicz SB, Guzzo TJ. Survival benefit persists with delayed initiation of adjuvant chemotherapy following radical cystectomy for locally advanced bladder cancer. Urology 2019;132:143-9. doi:10.1016/j.urology.2019.05.038

28 Booth CM, Siemens DR, Peng Y, Tannock IF, Mackillop WJ. Delivery of perioperative chemotherapy for bladder cancer in routine clinical practice. Ann Oncol 2014;25:1783-8. doi:10.1093/annonc/mdu204

29 Sanford RA, Lei X, Barcenas CH, et al. Impact of time from completion of neoadjuvant chemotherapy to surgery on survival outcomes in breast cancer patients. Ann Surg Oncol 2016;23:1515-21. doi:10.1245/s10434-015-5020-3

30 Gagliato D de Melo, Gonzalez-Angulo AM, Lei X, et al. Clinical impact of delaying initiation of adjuvant chemotherapy in patients with breast cancer. J Clin Oncol 2014;32:735-44. doi:10.1200/ JCO.2013.49.7693

31 Hershman DL, Wang X, McBride R, Jacobson IS, Grann VR, Neugut Al. Delay of adjuvant chemotherapy initiation following breast cancer surgery among elderly women. Breast Cancer Res Treat 2006:99:313-21. doi:10.1007/s10549-006-9206-z

32 Hershman D, Hall MJ, Wang X, et al. Timing of adjuvant chemotherapy initiation after surgery for stage III colon cancer. Cancer 2006;107:2581-8. doi:10.1002/cncr.22316

33 Cheung WY, Neville BA, Earle CC. Etiology of delays in the initiation of adjuvant chemotherapy and their impact on outcomes for Stage II and III rectal cancer. Dis Colon Rectum 2009;52:1054-64. doi:10.1007/DCR.0b013e3181a51173

34 Bayraktar UD, Chen E, Bayraktar S, et al. Does delay of adjuvant chemotherapy impact survival in patients with resected stage II and III colon adenocarcinoma?Cancer 2011;117:2364-70. doi:10.1002/ cncr. 25720

35 Lima IS, Yasui Y, Scarfe A, Winget M. Association between receipt and timing of adjuvant chemotherapy and survival for patients with stage III colon cancer in Alberta, Canada. Cancer 2011;117:3833-40. doi:10.1002/cncr.25954

36 Becerra AZ, Aquina CT, Mohile SG, et al. Variation in delayed time to adjuvant chemotherapy and disease-specific survival in stage III colon cancer patients. Ann Surg Oncol 2017;24:1610-7. doi:10.1245/s10434-016-5622-4

37 Turner MC, Farrow NE, Rhodin KE, et al. Delay in adjuvant chemotherapy and survival advantage in stage III colon cancer. J Am Coll Surg 2018;226:670-8. doi:10.1016/j.jamcollsurg.2017.12.048

38 Xu F, Rimm AA, Fu P, Krishnamurthi SS, Cooper GS. The impact of delayed chemotherapy on its completion and survival outcomes in stage II colon cancer patients. PLoS One 2014;9:e107993. doi:10.1371/journal.pone.0107993

39 Massarweh NN, Haynes AB, Chiang YJ, et al. Adequacy of the National Quality Forum's colon cancer adjuvant chemotherapy quality metric: is 4 months soon enough?Ann Surg 2015;262:312-20. doi:10.1097/SLA.0000000000000859

40 Booth CM, Shepherd FA, Peng Y, et al. Time to adjuvant chemotherapy and survival in non-small cell lung cancer: a population-based study. Cancer 2013;119:1243-50. doi:10.1002/cncr.27823

41 Salazar MC, Rosen JE, Wang Z, et al. Association of delayed adjuvant chemotherapy with survival after lung cancer surgery. JAMA Oncol 2017;3:610-9. doi:10.1001/jamaoncol.2016.5829

42 Hébert-Croteau N, Freeman CR, Latreille J, Rivard M, Brisson J. A population-based study of the impact of delaying radiotherapy after conservative surgery for breast cancer. Breast Cancer Res Treat 2004:88:187-96. doi:10.1007/s10549-004-0594-7

43 Jhawar S, Hathout L, Elshaikh MA, Beriwal S, Small W, Mahmoud O. Adjuvant chemoradiation therapy for cervical cancer and effect of timing and duration on treatment outcome. Int J Radiat Oncol Biol Phys 2017:98:1132-41. doi:10.1016/j.jirobp.2017.03.045

44 Sharma S, Bekelman J, Lin A, et al. Clinical impact of prolonged diagnosis to treatment interval (DTI) among patients with oropharyngeal squamous cell carcinoma. Oral Oncol 2016;56:17 24. doi:10.1016/i.oraloncology.2016.02.010

45 Harris JP, Chen MM, Orosco RK, Sirjani D, Divi V, Hara W. Association of survival with shorter time to radiation therapy after surgery for US patients with head and neck cancer. JAMA Otolaryngol Head Neck Surg 2018;144:349-59. doi:10.1001/jamaoto.2017.3406

46 Chen YP, Mao YP, Zhang WN, et al. Prognostic value of wait time in nasopharyngeal carcinoma treated with intensity modulated radiotherapy: a propensity matched analysis. Oncotarget 2016;7:14973-82. doi:10.18632/oncotarget.7789

47 Gupta S, King WD, Korzeniowski M, Wallace DL, Mackillop WJ. The Effect of Waiting Times for Postoperative Radiotherapy on Outcomes for Women Receiving Partial Mastectomy for Breast Cancer: a Systematic Review and Meta-Analysis. Clin Oncol 2016;28:739-49. doi:10.1016/j.clon.2016.07.010

48 Olivotto IA, Lesperance ML, Truong PT, et al. Intervals longer than 20 weeks from breast-conserving surgery to radiation therapy are associated with inferior outcome for women with earlystage breast cancer who are not receiving chemotherapy. / Clin Oncol 2009;27:16-23. doi:10.1200/JC0.2008.18.1891

49 Barton MB, Jacob S, Shafiq J, et al. Review of optimal radiotherapy utilisation rates. Ingham Institute, Collaboration for Cancer Outcomes Research and Evaluation, 2013, http://tinyurl.com/pwkua34.

50 Davis C, Naci H, Gurpinar E, Poplavska E, Pinto A, Aggarwal A. Availability of evidence of benefits on overall survival and quality of life of cancer drugs approved by European Medicines Agency: retrospective cohort study of drug approvals 2009-13. BM/ 2017;359:j4530. doi:10.1136/bmj.j4530

51 Lievens Y, Audisio R, Banks I, Collette L, Grau C, Oliver K, et al. Towards an evidence-informed value scale for surgical and radiation oncology: a multi-stakeholder perspective. Lancet Oncol 2019;20:e112-23. doi:10.1016/S1470-2045(18)30917-3

52 Luengo-Fernandez R, Leal J, Gray A, Sullivan R. Economic burden of cancer across the European Union: a population-based cost analysis. Lancet Oncol 2013;14:1165-74. doi:10.1016/S1470 2045(13)70442-X

53 Dossa F, Chesney TR, Acuna SA, Baxter NN. A watch-and-wait approach for locally advanced rectal cancer after a clinical complete response following neoadjuvant chemoradiation: a systematic review and meta-analysis. Lancet Gastroenterol Hepatol 2017;2:501-13. doi:10.1016/S2468-1253(17)30074-2

54 Chen C-P, Kung P-T, Wang Y-H, Tsai W-C. Effect of time interval from diagnosis to treatment for cervical cancer on survival: a nationwide cohort study. PLoS One 2019;14:e0221946. doi:10.1371/journal. pone.0221946

55 Tsai CH, Kung PT, Kuo WY, Tsai WC. Effect of time interval from diagnosis to treatment for non-small cell lung cancer on survival: a national cohort study in Taiwan. BMJ Open 2020;10:e034351 doi:10.1136/bmjopen-2019-034351

56 The Royal College of Radiologists. The timely delivery of radical radiotherapy: guidelines for the management of unscheduled treatment interruptions. The Royal College of Radiologists. 2019 https://www.rcr.ac.uk/system/files/publication/field_publication files/bfco191_radiotherapy-treatment-interruptions.pdf

57 McMillan MT, Ojerholm E, Verma V, et al. Radiation treatment time and overall survival in locally advanced non-small cell lung cancer. Int J Radiat Oncol Biol Phys 2017;98:1142-52. doi:10.1016/j. ijrobp.2017.04.004

58 Girinsky T, Rey A, Roche B, et al. Overall treatment time in advanced cervical carcinomas: a critical parameter in treatment outcome. Int J Radiat Oncol Biol Phys 1993;27:1051-6. doi:10.1016/03603016(93)90522-W

59 NHS England. Clinical guide for the management of essential cancer surgery for adults during the coronavirus pandemic. Version 1, 7 April 2020. https://www.asgbi.org.uk/userfiles/file/covid19/c0239specialty-guide-essential-cancer-surgery-coronavirus-v1-70420.pdf

60 Achieving world-class cancer outcomes: a strategy for England 2015 2020. https://www.iccp-portal.org/system/files/plans/Strategy\%20 -Final.pdf

61 van Dijk LV, Van den Bosch L, Aliabar P, et al. Improving automatic delineation for head and neck organs at risk by Deep Learning Contouring Radiother Oncol 2020;142:115-23. doi:10.1016/j.radonc.2019.09.022

62 Urbach DR, Martin D. Confronting the COVID-19 surgery crisis: time for transformational change. CMAI 2020;192:E585-6. doi:10.1503/ cmaj. 200791

63 Kim RH, Kavanaugh MM, Caldito GC. Laparoscopic colectomy for cancer: Improved compliance with guidelines for chemotherapy and survival. Surgery 2017;161:1633-41. doi:10.1016/j.surg.2016.11.024

64 Boyle JM, Kuryba A, Cowling TE, et al. Determinants of Variation in the Use of Adjuvant Chemotherapy for Stage III Colon Cancer in England. Clin Oncol (R Coll Radiol) 2020;32:e135-44. doi:10.1016/j. clon.2019.12.008

Web appendix: Appendices 